\title{
ESTUDO REOLÓGICO DA POLPA DE JUÇARA (Euterpe edulis Mart) EM FUNÇÃO DA TEMPERATURA E TEOR DE SÓLIDOS SOLÚVES
}

\author{
I. I. S. GUIDA ${ }^{1}$, H. A. VILLA-VÉLEZ¹, A. A. SANTANA¹, D. R. M. SAMPAIO1 \\ ${ }^{1}$ Universidade Federal Do Maranhão, Departamento de Engenharia Química \\ E-mail para contato: italoiury15@gmail.com
}

\begin{abstract}
RESUMO - A indústria de processamento de frutas exóticas tem crescido rapidamente no Brasil. A Juçara (Euterpe edulis Mart.) é abundante no território maranhense e apreciada para a produção de vários produtos. Este trabalho tem como objetivo estudar o comportamento reológico da polpa de juçara a diferentes temperaturas e concentrações. Para isto, análises reológicas foram realizadas para a polpa in natura e polpa diluída a $4^{\circ}$ Brix através de um reômetro modelo DV-II + Pro, Brookfield, USA. Observou-se que a viscosidade aparente diminuiu com o aumento da taxa de deformação e da temperatura. Também foi observada uma relação diretamente proporcional entre a viscosidade e a concentração de sólidos solúveis. O índice de comportamento do fluido calculado a partir do modelo da Lei da potência foi menor que 1 , característica de um fluido pseudoplástico. Finalmente, observou-se a dependência do índice de consistência do fluido com a temperatura, que diminui com o aumento da mesma. A energia de ativação calculada pelo modelo de Arrhenius foi de 16,095 e 9,585 kJ/gmol para a polpa in natura e diluída, respectivamente.
\end{abstract}

\section{INTRODUÇÃO}

A Juçara (Euterpe edulis Mart.) também conhecida como palmito-doce é bastante encontrada na Mata Atlântica e também no cerrado, perto dos cursos dos rios e matas úmidas, da Bahia ao Rio Grande do Sul e no Maranhão facilitando o contado direto com o fruto. Além de ter um sabor bastante apreciado, trata-se de um fruto refrescante, energético e rico em lipídios, vitamina A, ferro e água (MORTARA e VALERIANO, 2001).

A reologia é uma ciência que surgiu no início do século XX e tem como objetivo estudar a deformação e o escoamento dos materiais. O estudo reológico prediz a força necessária para causar uma deformação ou escoamento de um corpo resultado da aplicação de um dado sistema de forças que podem ser de compressão, tração ou cisalhamento. Com isso manipular produtos derivados das frutas na indústria requer o uso da polpa da fruta, na qual é submetida aos processos industriais como bombeamento, agitação, transporte através de tubulações, evaporação entre outros. Para que essas operações unitárias sejam economicamente viáveis, é indispensável o conhecimento das propriedades físicas e químicas da polpa do fruto a ser processado. O comportamento reológico por sua vez, destaca-se por ser útil não apenas como medida de qualidade, mas também em projetos, avaliação e operação de equipamentos processadores de alimentos (SILVA et al, 2005). 
Este trabalho tem como objetivo estudar o comportamento reológico da polpa de Juçara Maranhense em função da temperatura e do teor de sólidos solúveis, visando seu melhor processamento e aproveitamento a nível industrial.

\section{MATERIAIS E MÉTODO}

A polpa de Juçara foi adquirida no mercado local da cidade de São Luís, MA. O fruto foi moído no momento da compra e a polpa foi preparada e colocada dentro de sacos de polietileno de $100 \mathrm{~g}$. Após isso, a polpa foi armazenada em um freezer vertical a $-20^{\circ} \mathrm{C}$ (modelo CRM33, Consul, Brasil) para posterior uso na pesquisa.

\subsection{Caracterização reológica e modelagem matemática}

O comportamento reológico da polpa de juçara foi analisado nas temperaturas de 10, 35 e $60{ }^{\circ} \mathrm{C}$ na sua forma integral e diluída com um teor de sólidos solúveis de 4 ( ${ }^{\circ}$ Brix). As medidas reológicas foram realizadas em um reômetro (modelo DV-II + Pro, Brookfield, USA), utilizando um spindle LV3(63). Um banho termostático (modelo ALB 250 C, Tecnal, Brasil) foi utilizado para manter as temperaturas durante os experimentos. A análise reológica para cada temperatura foi realizada na taxa de deformação crescente de 0,33 a 3,33 $\mathrm{s}^{-1}$. Para cada temperatura foi empregada uma amostra de Juçara adequada à temperatura de estudo.

Para obtenção dos parâmetros reológicos e para determinação do seu comportamento, utilizou-se o modelo da Lei da Potência (Equação 1) e a viscosidade para fluidos não newtonianos (Equação 2).

$$
\begin{aligned}
& \tau=k \gamma^{n} \\
& \mu=k \gamma^{n-1}
\end{aligned}
$$

onde $\tau$ é a tensão de cisalhamento (Pa), $\mu$ é a viscosidade dinâmica (Pa.s), $n$ é o índice de comportamento (adimensional), $\gamma$ é a taxa de deformação $\left(\mathrm{s}^{-1}\right) \mathrm{e}, k$ é o índice de consistência (Pa.s) (GENOVESE e LOZANO, 2007). Além disso, através deste modelo foi estudado o efeito da temperatura na concentração de sólidos através da relação de Arrhenius, como mostrado na Equação 3.

$$
\eta=A_{0} \exp \left(\frac{E_{a}}{R(T+273.15)}\right)
$$

onde $A_{0}$ é uma constante empírica, $\eta$ é a viscosidade aparente (mPa.s), $E_{a}$ é a energia de ativação (J/mol), $R$ é a constante universal dos gases $(8.314 \mathrm{~J} / \mathrm{mol} \mathrm{K})$ e $T$ é a temperatura $\left({ }^{\circ} \mathrm{C}\right)$.

A modelagem matemática foi realizada com o auxílio da ferramenta Solver do software Microsoft Excel (Microsoft, Redmond, EUA). O coeficiente de correlação e o erro médio relativo $(M R E, \%)$ descrito na Equação 4, foram usados para validar o ajuste do modelo.

$$
M R E=\frac{100}{n} \sum_{i=1}^{m} \frac{\left|Y_{i}-Y_{i}^{*}\right|}{Y_{i}^{*}}
$$


onde, $Y$ e $Y^{*}$ representam os valores experimentais e calculados, respectivamente, $m$ é o número de valores experimentais.

\section{RESULTADOS E DISCUSSÃO}

\subsection{Reogramas para a polpa de juçara in natura e diluída a $4\left({ }^{\circ}\right.$ Brix $)$}

Na Figura 1 estão apresentados os dados da viscosidade aparente experimental e teórica calculada a partir da Equação 2 para a polpa de Juçara in natura e diluída a $4\left({ }^{\circ}\right.$ Brix $)$. A viscosidade aparente está em função da taxa de cisalhamento para as temperaturas de 10, 35 e $60^{\circ} \mathrm{C}$.

Figura 1 - Viscosidade aparente para a polpa de Juçara in natura a) e diluída b).
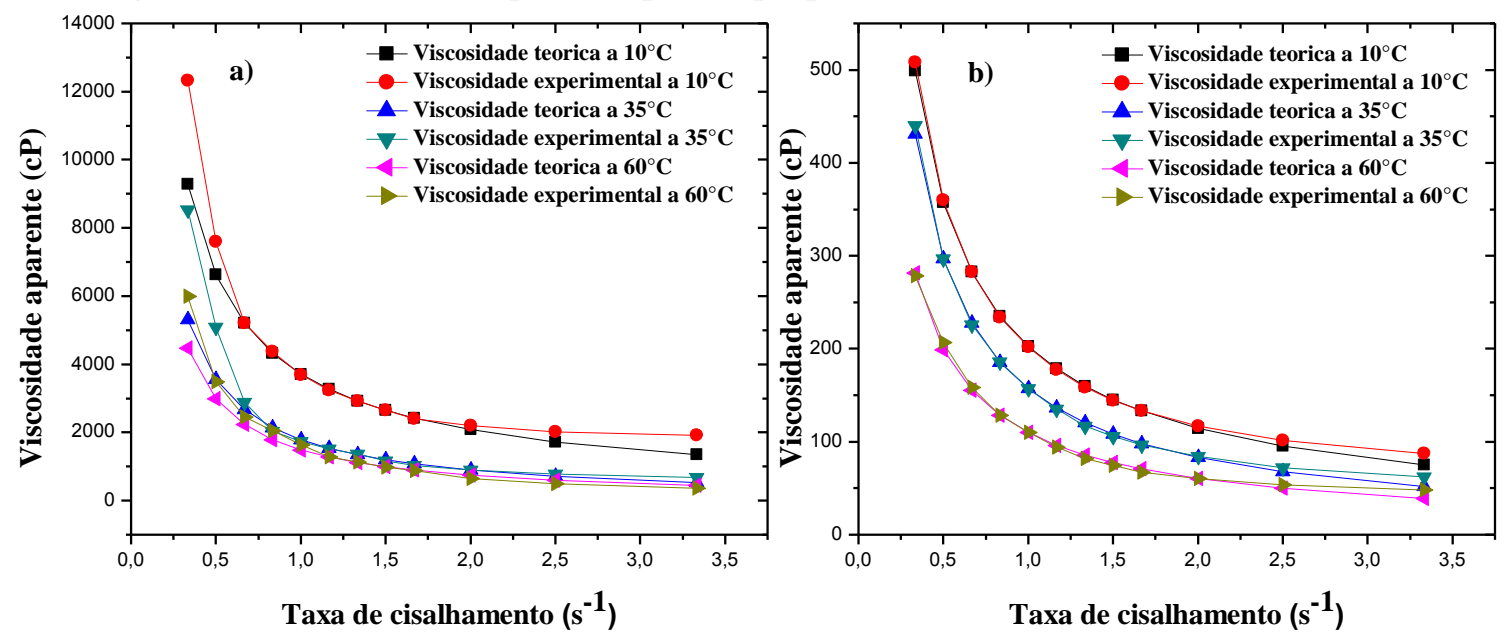

Observamos na Figuras 1 que o comportamento da viscosidade aparente não variou muito na polpa de Juçara a $4\left({ }^{\circ}\right.$ Brix). Já na polpa in natura, pode-se observar uma maior variação da viscosidade aparente com o aumento da taxa de deformação. Em ambos os casos (polpa in natura e a $4{ }^{\circ}$ Brix), percebe-se o bom ajuste do modelo da Lei da Potência aos dados experimentais apesar do reograma para a polpa in natura ter se comportado um pouco menos uniforme que para a polpa diluída.

O decréscimo na viscosidade aparente significa que a resistência das amostras ao escoamento e a energia requerida para mantê-los a uma alta taxa de deformação é reduzida. Reduções de viscosidade com o aquecimento também são reportadas em VIDAL et al., (2000) trabalhando com polpa de manga. A viscosidade aparente também depende da temperatura. As menores viscosidades foram observadas na temperatura mais altas $\left(60{ }^{\circ} \mathrm{C}\right)$. Os coeficientes de ajuste (n, k), a temperatura de trabalho $(\mathrm{T})$ e os parâmetros estatísticos ( $\left.\mathrm{R}^{2}, \mathrm{MRE}\right)$ estão apresentados na Tabela 1.

\subsection{Modelagem dos parâmetros reológicos e análise matemática para a polpa de Juçara}

Com o bom ajuste dos reogramas ao modelo da lei da potência no qual observamos um bom coeficiente de correlação $\left(\mathrm{R}^{2}\right)$, onde em todas as temperaturas o mesmo foi maior que 
0,928 e o MRE percentual menor que $10 \%$, com exceção apenas das temperaturas de 35 e $60^{\circ} \mathrm{C}$ para a polpa in natura, cujo MRE $=10,10 \%$ e $10,52 \%$, podemos então usar a modelagem matemática para calcular os parâmetros reológicos com uma boa precisão e então classificar nosso fluido quanto a sua natureza.

Ainda ao MRE, podemos associar esse erro a convecção natural que age sobre o banho termostático durante os experimentos, no qual mesmo sendo pequena a variação de temperatura devido a bom controle do banho, ela ainda interfere nas leituras do reômetro. Com isso, podemos então calcular o índice de comportamento do fluido e o índice de consistência que estão apresentados na Tabela 1.

Tabela 1 - Parâmetros reológicos do modelo da lei da potência para a polpa de Juçara in natura e diluída a 4 ( ${ }^{\circ}$ Brix), onde $\mathrm{k}$ é o índice de consistência $\left(\mathrm{mPa} \cdot \mathrm{s}^{\mathrm{n}}\right), n$ é o índice de comportamento do fluido (adimensional) e MRE é o erro médio relativo (\%)

\begin{tabular}{cccccc}
\hline Polpa & $\mathbf{T}\left({ }^{\circ} \mathbf{C}\right)$ & $\mathbf{k}\left(\mathbf{m P a . s} \mathbf{s}^{\mathbf{n}}\right)$ & $\boldsymbol{n}$ & $\mathbf{R}^{\mathbf{2}}$ & MRE (\%) \\
\hline In natura & 10 & 3716,54 & 0,170 & 0,966 & 7,47 \\
& 35 & 1793,06 & 0,010 & 0,964 & 10,10 \\
& 60 & 1495,86 & 0,001 & 0,993 & 10,56 \\
Diluída (4 & & & & & \\
& 10 & 202,66 & 0,179 & 0,998 & 2,31 \\
& 35 & 157,16 & 0,080 & 0,998 & 2,93 \\
& 60 & 109,99 & 0,143 & 0,996 & 4,07 \\
\hline
\end{tabular}

Analisando os parâmetros reológicos, podemos então dizer que a polpa de Juçara Maranhense integral e diluída apresentou um comportamento de fluido pseudoplástico nãoNewtoniano já que o índice de comportamento foi menor que 1 . O mesmo comportamento pseudoplástico foi encontrado em um estudo anterior realizado com a polpa de cenoura pasteurizada (VANDRESEN et al., 2009). Ainda na Tabela 1, percebemos que o índice de comportamento do fluido não variou muito, contudo, o índice de consistência foi diminuindo com aumento da temperatura e com a diminuição da concentração de sólidos solúveis. Observamos então que o (k) é diretamente ligado a concentração de sólidos solúveis e a temperatura, onde, nota-se claramente sua dependência. Observamos então uma manutenção do comportamento pseudoplástico, já que o $n$ não mudou significativamente da polpa in natura para a diluída.

Podemos perceber portanto que de modo geral, as polpas de frutas mostram um comportamento pseudoplástico. Esses fluidos apresentam uma diminuição da tensão de cisalhamento com o aumento da taxa de cisalhamento, que depende principalmente da orientação/alinhamento das moléculas ou partículas na direção do fluxo (VRIESMANN, 2008).

\subsection{Efeito da temperatura no comportamento reológico e avaliação da energia de ativação pela equação de Arrhenius}

Para descrever o comportamento de escoamento da polpa de Juçara em função da temperatura utilizou-se a equação de Arrhenius, Equação 3. Linearizando a Equação 3 e aplicando a função ln em ambos os lados da equação, obtivemos os valores experimentais de ln (viscosidade aparente) versus temperatura $\left(\mathrm{K}^{-1}\right)$. Podemos assim observar as taxas de 
deformação constantes na Figura 2 para a polpa in natura e diluída. Podemos ainda observar os parâmetros obtidos nos ajustes na Tabela 2.

Figura 2 - Efeito da temperatura para a polpa de juçara a) in natura e b) diluída em diferentes taxas de deformação.
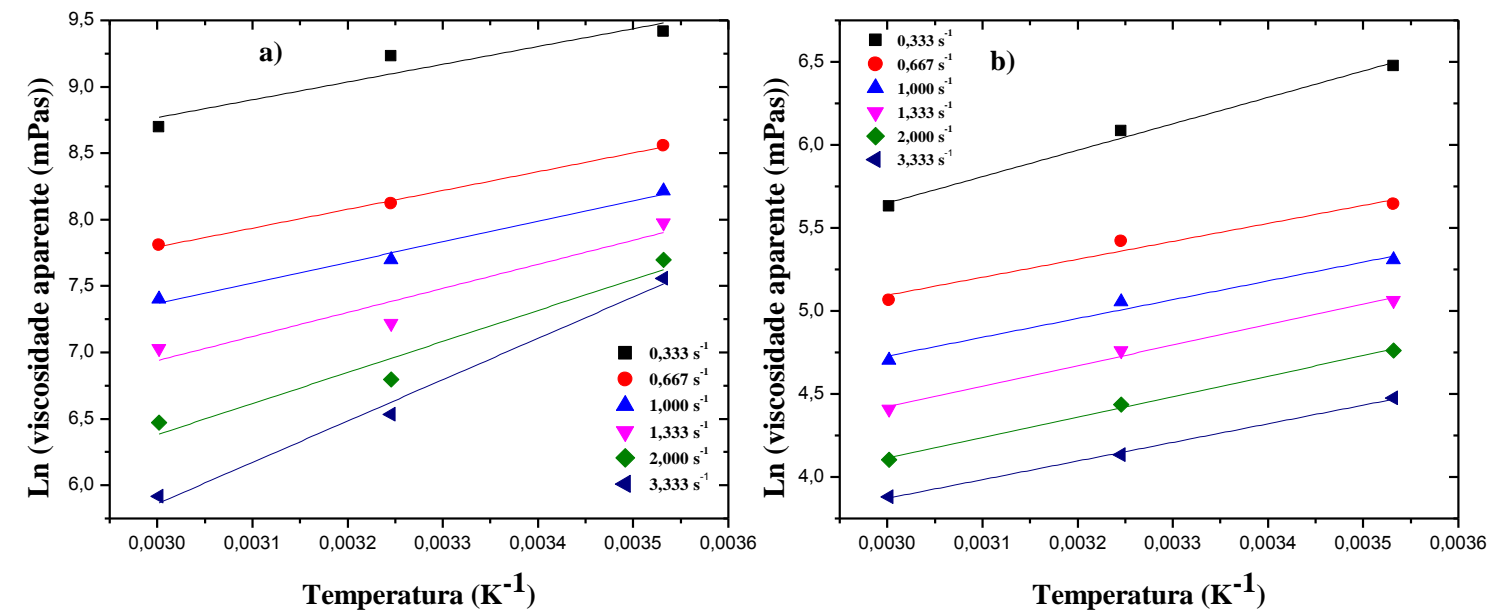

Observa-se na Tabela 2 que a energia de ativação foi em média igual a 16,095kJ/gmol $\left(\mathrm{R}^{2}=0,986\right)$ para a polpa in natura e $9,585 \mathrm{~kJ} / \mathrm{gmol}\left(\mathrm{R}^{2}=0,970\right)$ para a polpa diluída e estão de acordo com o encontrado na literatura para polpas de fruta.

Tabela 2 - Parâmetros da equação de Arrhenius há diferentes taxas de deformação para a polpa de Juçara in natura e diluída

\begin{tabular}{ccccc}
\hline Polpa & $\begin{array}{c}\text { Taxa de deformação } \\
\left(\mathbf{s}^{-\mathbf{1}}\right)\end{array}$ & $\mathbf{E a} / \mathbf{R}$ & $\mathbf{R}^{\mathbf{2}}$ & $\begin{array}{c}\text { Ea } \\
(\mathbf{k J} / \mathbf{g m o l})\end{array}$ \\
\hline \multirow{3}{*}{ In natura } & 0,333 & 1356,7 & 0,99 & 11279,6038 \\
& 0,667 & 1431,9 & 0,98 & 11904,8166 \\
& 1,000 & 1567,4 & 0,98 & 13031,3636 \\
& 1,333 & 1811,3 & 0,99 & 15059,1482 \\
Média & 2,000 & 3111,5 & 0,99 & 25869,011 \\
& 3,333 & 2336,7 & 0,99 & 19427,3238 \\
Diluída (4' ${ }^{\circ}$ Brix) & & $\mathbf{1 9 3 5 , 9 1}$ & $\mathbf{0 , 9 8 6}$ & $\mathbf{1 6 , 0 9 5}$ \\
& 0,333 & 1114,8 & 0,98 & 9268,4472 \\
& 0,667 & 1081,5 & 0,93 & 8991,591 \\
& 1,000 & 1132,1 & 0,95 & 9412,2794 \\
& 1,333 & 1231,0 & 0,98 & 10234,534 \\
Média & 2,000 & 1236,3 & 0,99 & 10278,5982 \\
& 3,333 & 1121,8 & 0,99 & 9326,6452 \\
\hline
\end{tabular}

Os resultados indicam uma diminuição da energia de ativação da polpa de Juçara quando a concentração de sólidos solúveis é menor. Esse comportamento era esperado, pois quanto maior a concentração, maior será a energia mínima necessária para fazer com que o fluido escoe. Isso acontece porque em concentrações elevadas da polpa, existem quantidades de compostos como proteínas, vitaminas entre outros nutrientes que interagem entre si com maior facilidade quando comparado a polpa diluída através de cargas elétricas, o que faz com que a viscosidade do fluido aumente devido ao empacotamento das moléculas. 


\section{CONCLUSÃO}

Analisando os resultados obtidos a partir do estudo da polpa de Juçara, pode-se concluir que a viscosidade aparente e a energia de ativação se comportaram de forma semelhante ao encontrado na literatura para polpa de fruta.

Observa-se ainda que os dados experimentais da tensão de cisalhamento e da taxa de deformação da polpa de Juçara in natura e diluída se ajustaram bem ao modelo da Lei da Potência, o que indicou que tanto a polpa in natura, quanto a diluída, apresentaram comportamento de um fluido não-newtoniano do tipo pseudoplástico, no qual, só foi possível fazer essa classificação a partir da modelagem matemática em que o índice de comportamento do fluído foi menor que 1 .

Por último, observou-se que quando mais concentrada é a polpa de juçara, maior será a energia de ativação mínima para que o fluido em questão escoe. Portanto, foi possível cumprir os objetivos desse estudo quanto ao estudo reológico e dependência da temperatura e concentração de sólidos solúveis para a polpa de Juçara.

\section{REFERÊNCIAS}

COSTA, F. Q. Viabilidade de uso de um misturador para a análise de propriedades reológicas de produtos alimentícios. 115f. Dissertação (Mestrado em Agronomia) - Universidade Estadual Paulista, Botucatu-SP, 2007.

GENOVESE, D.B.; LOZANO, J.E.; RAO, M.A. The rheology of colloidal and noncolloidal food dispersions, Journal of Food Science, v. 72, p. 11-20, 2007.

MORTARA, M. O.; VALERIANO, D. M. Modelagem da distribuição potencial do palmiteiro (Euterpe edulis martius) a partir de variáveis topográficas. In: Simpósio brasileiro de sensoriamento remoto, 10. (SBSR)., 2001, Foz do Iguaçu, São José dos Campos: INPE, 2001.

SILVA, F.C.; GUIMARÃES, D. H. P.; GASPARETTO, C.A. Reologia do suco de acerola: efeitos da concentração e temperatura. Ciência e Tecnologia de Alimentos, Campinas, v. 25, n.1, p.121-126, jan.-mar., 2005.

VANDRESEN, S.; MARA, G.N. Q.; JOSE, A.R.S.; DACHAMIR, H. Temperature effect on the rheological behavior of carrot juices, Journal of Food Engineering, v. 92, n. 3, p.269-274, Jun. 2009.

VIDAL, J.R.M.B.; GASPARETTO, C.A.; GRANDIN, A. Efeito da temperatura no comportamento reológico da polpa de manga. Revista Ciências Exatas e Naturais, v.1, n.2, p.69-76, 2000.

VRIESMANN, L. C. Extração, caracterização e aspectos reológicos de polissacarídeos da polpa dos frutos de theobroma grandiflorum. Dissertação (Mestrado em Bioquímica de alimentos) - Universidade Federal do Paraná. 2008. 